\title{
EVALUACIÓN Y CORRELACIÓN DE COMPONENTES DE RENDIMIENTO EN LÍNEAS AVANZADAS DE ARVEJA Pisum sativum CON GEN AFILA
}

\section{EVALUATION AND CORRELATION OF YIELD COMPONENTSIN ADVANCED LINES OF PEA Pisum sativum WITH AFILA GENE}

\author{
Darío Pantoja G.; Keily Muñoz Z. ${ }^{1}$; Oscar Checa C. ${ }^{2}$
}

Fecha de recepción: Mayo 09 de 2014

Fecha de aceptación: Agosto 20 de 2014

\begin{abstract}
RESUMEN
En el departamento de Nariño las variedades de arveja Pisum sativum que se cultivan son prediminantemente plantas de porte alto y abundante ramificación, que requieren sistemas de tutorado costosos para evitar el volcamiento y la proliferación de enfermedades. Producir cambios en la arquitectura de la planta utilizando variedades con gen afila que remplaza hojas por zarcillos, puede contribuir a desarrollar sistemas de tutorado mas simples y a disminuir el uso de fibras de polipropileno para el amarre reduciendo los costos de producción del cultivo. Esta investigación se realizó en la granja Lope perteneciente al Servicio Nacional de Aprendizaje (SENA), Pasto-Colombia, con el objetivo de evaluar genotipos de arveja P. sativumy contribuir a la obtención de nuevas variedades con gen afila para mejorar la competitividad de la leguminosa en el departamento de Nariño. Se empleó un diseño de bloques completamente al azar (BCA) con tres repeticiones y 22 tratamientos, durante el semestre A del 2013. Los tratamientos consistieron en 20 lineas F5 de arveja volubles semiafilas obtenidas en la Universidad de Nariño y dos variedades testigo no afilas (Andina y Sindamanoy). Se obtuvo diferencias entre genotipos en las variables número de granos por vaina, peso de vaina verde, peso de grano verde por vaina y rendimiento. El rendimiento correlacionó genotípicamente con número de granos por vaina, peso de vaina verde y peso de grano verde por vaina. En el análisis de sendero la mayor contribución indirecta positiva,
\end{abstract}

1 Estudiantes Ingeniería Agronómica, Universidad de Nariño. Pasto. Colombia

2 Docente Tiempo Completo, Ph.D. Universidad de Nariño. Pasto. Colombia. cicagrarias@hotmail.com 
fue el peso de grano por vaina verde vía peso de vaina verde, por lo tanto estas variables e se usaroncomo criterio de selección que permitió identificar como promisorias a las líneas UDENAR11, UDENAR12, UDENAR14, UDENAR19 y UDENAR20.

Palabras clave: Arveja, análisis de sendero, correlación genotípica.

\begin{abstract}
In the department of Nariño, the cultivated varieties of pea Pisum sativum L. are predominantly those with tall plants and abundant branching, which require expensive tutoring systems to prevent tipping and the spread of diseases. Producing changes in the plant's architecture, using pea varieties with the afil gene, which replaces leaves for tendrils, may help the development of simpler tutoring systems and decrease the use of polypropylene fibers used to tie the plant, reducingcrop production costs. This research was conducted at the Lope farm belonging to the Servicio Nacional de Aprendizaje (SENA) in Pasto-Colombia,with the goal of evaluatinggenotypes of pea Pisum sativum L. and contributing to the development of new varieties with the afila gene to improve the competitiveness of the legume in the department of Nariño. A randomized complete block design with 3 replicas and 22 treatments was conductedduringsemester A of 2013. The treatments consisted of 20 F5 lines of climbing semi-leafless peas obtained at the Universidad de Nariño, and two control non-afila varieties (Andina and Sindamanoy). We observed differences between genotypes in terms of the variables: number of grains per pod, green pods weight, weight of green grains per pod, and grain yield. Yield correlated genotypically with the number of grains per pod, green pod weight, and weight of green grains per pod. Path analysis showed that the highest indirect positive contribution wasmade by the weight of the grain per green pod throughthe green pod weight; therefore, these were used as selection criteria that allowed the identification of UDENAR11, UDENAR12, UDENAR14, UDENAR19, and UDENAR20 as promising lines.
\end{abstract}

Keywords: Pea, path analysis, genotypic correlation.

\section{INTRODUCCIÓN}

En Colombia la arveja Pisum sativum L, después del fríjol Phaseolus vulgaris L., es la leguminosa de mayor importancia. Esta ha sido un factor estabilizador de la economía de los pequeños productores de las zonas andinas, y ha contribuido a su seguridad alimentaria. Se estima que de este cultivo dependen más de 26.000 productores y genera alrededor de 2,3 millones de jornales (FENALCE, 2010). Se cultiva en catorce departamentos y su producción se concentra en Nariño con 48264 toneladas (55,2\% de la producción nacional), seguido de Cundinamarca $(20,4 \%)$ y Boyacá $(12 \%)$ (ENA, 2014).

Las características importantes a tener en cuenta para la elección de variedades apropiadas de arveja son la productividad y la tolerancia varietal al volcamiento. Previamente a la consideración de la altura de la variedad, es imprescindible conocer el tipo de hoja. Se puede encontrar variedades con foliolos abundantes y zarcillos 
poco desarrollados, afilas (sin foliolos y zarcillos muy desarrollados) o variedades semiafilas (intermedio entre los dos) (ITGA, 2003). En general se puede decir que a mayor desarrollo del zarcillo, la resistencia al volcamiento es mayor (Wang et al., 2003). El gen afila es recesivo y conduce a la expresión fenotípica solamente si se presenta en combinación homocigota recesiva (afaf). Se expresa con la transformación de hojas en zarcillos (Kujala, 1953; Goldenberg, 1965; Hedley y Ambrose, 1981; Marx, 1987; Prohens y Nuez, 2007; Singhetal., 2013). En el mundo se han reportado varios lanzamientos de variedades de arveja con gen afila, en diferentes años, entre las cuales se encuentran: Solara en 1986, Alex y Choque en 1989, Amadeus en 1992, Charleston y Astuceen 1993 (Mera et al., 1998), Rampart y Stampede en 1990 (Department of Horticultural Science, 1999), Brisca-INIA en 1996 (Mera et al., 1996), Golijat en 2001 (Djordjevic et al., 2001). Gracias a su follaje con gen afila, las plantas resisten al volcamiento temprano que afecta las variedades de follaje convencional. La ausencia del volcamiento temprano es importante porque mantiene la aireación (Giaconi, 2004), lo cual favorece la sanidad del follaje (Mera et al., 1996). Además, estas variedades tienden a incrementar su productividad respecto a las variedades no afilas (Jannink et al., 1996; Mihailovic, 2008) y a ser más eficientes en el uso del agua puesto que hay menos pérdida por transpiración al tener menos superficie foliar, haciéndolas más tolerantes a la sequía (Cousin et al., 1986; Martin et al., 1994).

En los procesos de evaluación y selección de genotipos mejorados es necesario identificar caracteres que tienen importancia sobre el rendimiento y que puedan ser utilizados como criterios de selección. El análisis de sendero, es la técnica estadística utilizada para examinar las relaciones causales entre un conjunto de variables independientes y la va- riable dependiente, (Hazard, 2005; Moses, 2006; Garson, 2012; Sarutayophat, 2012) ayudando a los fitomejoradores a la selección de genotipos de mejor desempeño.

Las variedades mejoradas de arveja de mayor uso en Nariño (Colombia), son de porte alto con follaje normal, que requieren la implementación de tutorado vertical y horizontal, para evitar el volcamiento y la proliferación de enfermedades foliares, aumentando los costos de producción que ascienden a $\$ 8 ` 300.000$ (pesos colombianos) por hectárea (2014) de los cuales el 52\% corresponden a los gastos por tutorado (Checa, 2014). Encontrar genotipos de arveja voluble con gen afila que logren igualar o superar los rendimientos de las variedades de mayor uso en la región, pueden ser una nueva alternativa productiva, que permita reducir el acame de las plantas y disminuir los costos por tutorado, mejorando la competitividad del cultivo.

El objetivo de la presente investigación fue evaluar el comportamiento agronómico de 20 líneas de arveja volubles con gen afila y determinar el grado de asociación que existe entre sus componentes de rendimiento, para contribuir al mejoramiento genético de la arveja (P.sativum) en el departamento de Nariño.

\section{MATERIALES Y MÉTODOS}

\section{Descripción del área de estudio}

El trabajo se desarrolló en la Granja Lope perteneciente al Servicio Nacional de Aprendizaje (SENA), Pasto-Colombia, ubicada a $2633 \mathrm{msnm}$ $1^{\circ} 12^{\prime}$ latitud N y $77^{\circ} 15^{\prime}$ longitud W con una temperatura promedio de $13^{\circ} \mathrm{C}$ y una precipitación promedio anual de $700 \mathrm{~mm}$. 


\section{Material y diseño experimental}

El programa de mejoramiento genético del cultivo de arveja de la Universidad de Nariño, desarrolló líneas avanzadas afila resultantes del cruzamiento de las variedades volubles mejoradas de hojas normales Andina, Sindamanoy y San Isidro con genotipos arbustivos portadores del gen afila ILS3568, ILS3575 y Dove. Teniendo en cuenta la condición recesiva del gen afila, su manifestación fenotípica se observó en la cuarta parte de las plantas de la generación F2. Este material se sembró en F3, seleccionándose 105 plantas individuales que generaron igual número de familias afila en F4, evaluadas cualitativamente por sanidad, carga y rendimiento, lo cual permitió seleccionar 52 líneas afila para la generación F5.

Estas líneas se evaluaron preliminarmente por periodo vegetativo y componentes de rendimiento aplicando un índice de selección que permitió la identificación de las 20 de mejor desempeño constituyendo el material genético de la presente investigación. Las líneas fueron identificadas con un código alfanumérico en el cual las seis primeras letras correspondieron a UDENAR acompañadas de los números del 1 al 20 y como testigos, las variedades regionales no afilas Andina y Sindamanoy.

Se evaluaron las variables: altura de la planta (ALT) medida en centímetros (cm), número de vainas por planta (NVP), número de granos por vaina (NGV), peso de vaina llena en verde (PVV) medida en gramos $(\mathrm{g})$, peso de grano verde por vaina (PGVV) medida en gramos (g) y rendimiento en vaina verde (REND) en $t \mathrm{ha}^{-1}$.

Las líneas fueron evaluadas en un ensayo formal de rendimiento utilizando un diseño de bloques completos al azar (BCA) con tres repeticiones. El tamaño de la unidad experimental fue de 2 sur- cos de $3 \mathrm{~m}$ de largo con distancia entre surcos de $1 \mathrm{~m}$ para un área de $6 \mathrm{~m}^{2}$. El área útil por parcela fue de 5,6 $\mathrm{m}^{2}$. El manejo agronómico se realizó con base a las recomendaciones de Sañudo et al., (1999).

\section{Análisis estadístico}

Los datos obtenidos fueron sometidos a un análisis de varianza y prueba de comparación de promedios de Tukey usando el programa GENES (Cruz, 2004). En el programa GENES, se aplicaron las fórmulas clásicas de correlación genética como la describe Falconer (1986) y estima Ceballos (2003):

$$
r G_{(X Y)}=\operatorname{COVG}_{(X Y)} / S_{G}(x) \cdot S_{G}(y)
$$

En donde: $\mathrm{rG}_{(\mathrm{XY})}$ y $\mathrm{COVG}_{(\mathrm{XY})}$ corresponden a la correlación y covarianza genética entre los caracteres $X$ y $Y ; S_{(x)}$ y $S_{(y)}$ son la desviación estándar genética de $X$ y $Y$. La significancia estadística de los coeficientes de correlación se estableció mediante una prueba de T, dada por el siguiente modelo:

$$
T_{c}=\frac{r \sqrt{(n-2)}}{\sqrt{\left(1-r^{2}\right)}}
$$

La T calculada (Tc): se comparó con una T tabular (Tt), al nivel de significancia seleccionando: 0,05 con $(n-2)$ grados de libertad. La regla de decisión fue: sí Tc $\geq \mathrm{Tt}$, entonces el valor de $\rho$ es estadísticamente diferente de cero.

Se realizó un análisis de sendero para el sistema REND como variable efecto $(Y)$ en función de las variables causa: $\mathrm{NGV}\left(\mathrm{X}_{1}\right), \operatorname{PVV}\left(\mathrm{X}_{2}\right), \mathrm{Y}$ PGVV $\left(X_{3}\right)$, empleando la matriz de correlaciones genotípicas entre tales variables para identificar los efectos directos e indirectos que participan en la correlación. 
La descomposición de los coeficientes de correlación de cada una de las variables causa con la variable efecto $\left(\mathrm{r}_{\mathrm{X}_{\mathrm{i}}}\right)$, en sus componentes efecto directo (Pi) y efecto indirecto (Ei), se realizó con base en la metodología usada por Espitia et al. (2008), permitiendo mediante el despeje de las siguientes ecuaciones, estimar los respectivos efectos indirectos de cada variable causa (Ei):

$$
\begin{gathered}
r X_{1} Y=P_{1}+E_{1}: \text { para } N G V \\
r X_{2} Y=P_{2}+E_{2}: \text { para PVV } \\
r X_{3} Y=P_{3}+E_{3}: \text { para PGVV }
\end{gathered}
$$

El coeficiente de sendero (h) debido a los efectos residuales o debido a otras variables no consideradas en el estudio, se estimó mediante la siguiente ecuación:

$$
h=\left[1-\left(P_{1} \cdot r X_{1} Y\right)-\left(P_{2} \cdot r X_{2} Y\right)-\left(P_{3} \cdot r X_{3} Y\right)\right]^{1 / 2}
$$

La regla de decisión para los coeficientes de correlación, se analizaron con base en la regla propuesta por Cohen y Holliday (1996), según los cuales se considera valores mayores en magnitud (alta correlación) cuando ( $|\mathrm{rG}|>0,50)$.

\section{RESULTADOS Y DISCUSIÓN}

El análisis de varianza (Tab. 1), presentó diferencias estadísticas significativas $(\mathrm{p}<0,05)$ entre genotipos para las variables número de granos por vaina $(\mathrm{NGV})$, peso de vaina verde (PVV), peso de grano verde por vaina (PGVV) y rendimiento en verde (REND). No se observaron diferencias estadísticas significativas entre líneas para las variables de altura de planta (ALT) y número de vainas por planta (NVP).

Es posible que por ser la altura de planta (ALT) uno de los criterios de evaluación preliminar, cuando de 52 líneas se llegó a la selección de las 20 mejores con las cuales se realizó el presente estudio, exista poca variación fenotípica en este carácter. En el mismo sentido el no encontrar diferencias estadísticas en el número de vainas por planta (NVP), puede asociarse conla selección cualitativa por carga realizada en la misma evaluación previa, la cual permitió a las 20 líneas ubicarse entre las de mejor comportamiento.

Los promedios de las líneas oscilaron entre 10 y 21 vainas por planta, siendo estadísticamente iguales a los obtenidos por los testigos Andina y Sindamanoy (sin gen afila) que alcanzaron promedios entre 16,33 y 19,67 vainas por planta.

De acuerdo con estos resultados, no hay evidencia que sugiera que la presencia del gen afila en las líneas evaluadas produzca reducción en el número de vainas por planta, puesto que los testigos no presentan el remplazo de hojas por

Tabla 1. Cuadrado medio para las variables altura (ALT), número de vainas por planta $(\mathrm{NVP})$, número de granos por vaina (NGV), peso de vaina verde (PVV), peso de grano verde por vaina (PGVV) y rendimiento (REND)

\begin{tabular}{lccccccc}
\hline Fuente de variación & G.L & ALT & NVP & NGV & PVV & PGVV & REND \\
\hline Genotipo & 21 & $149,06^{\text {ns }}$ & $20,47^{\text {ns }}$ & $1,15^{*}$ & $4,35^{*}$ & $1,48^{*}$ & $12,49^{*}$ \\
Bloques & 21 & $2409,02^{\text {ns }}$ & $73,27^{\text {ns }}$ & $0,17^{\text {ns }}$ & $2,08^{\text {ns }}$ & $0,40^{\text {ns }}$ & $35,62^{\text {ns }}$ \\
Error & 21 & 172,70 & 18,84 & 0,21 & 0,72 & 0,15 & 4,32 \\
R $^{2}$ & & 0,52 & 0,42 & 0,74 & 0,75 & 0,84 & 0,65 \\
C.V & & 9,32 & 26,45 & 7,16 & 12,38 & 9,46 & 26,00 \\
\hline
\end{tabular}

*Diferencias significativas $(\mathrm{p}<=0,05)$ 
zarcillos característico en las líneas con gen afila. En las evaluaciones de Mihailović et al. (2008), se encontró que los cultivares con gen afila se caracterizan por no tener una disminución de la actividad fotosintética y por tener los rendimientos incluso más altos en comparación con los cultivares de tipo hoja normal.

Lo anterior sugiere que las diferencias o similitudes entre genotipos con gen afila y sin gen afila para número de vainas, pueden estar más relacionadas con el transfondo genético de los genotipos evaluados que con la presencia del gen afila.

Por otra parte Engels et al., (1991), afirman que el número de vainas por planta para la especie $P$. satioum puede variar de 10 a 142, valor acorde a los obtenidos. Al respecto, Fierro (2012) encontró promedios de 6,27 hasta 51,73 vainas por planta. Por el contrario, los promedios encontrados por otros investigadores para este rasgo, en materiales afila, varían por debajo de los valores hallados en este estudio. Mera et al., (2007) obtuvo un rango de 8,25 y 9,70 vainas por planta mientras que Ligarreto et al., (2009) en materiales volubles no afila, reportan medias de 6,75 a 11,33 vainas por planta.

El coeficiente de variación para NVP en esta evaluación fue de 26,45\%, similar al reportado por Garzón y Gasca (1990) con un valor de $27,4 \%$ y Kumar et al. 2013) con 25,82\%, quienes lograron obtener diferencias entre los genotipos evaluados. Lo anterior sugiere mayor variabilidad genética entre las líneas evaluadas por dichos autores.

\section{Número de granos por vaina (NGV)}

El 86,4\% de los genotipos evaluados no presentan diferencias significativas con medias que oscilaron entre 5,70 y 7,11 granos por vaina (Tab.
2). Dentro de este grupo se encuentran los testigos Andina y Sindamanoy (6,73 y 7,00 granos por vaina respectivamente). Es importante resaltar los genotipos UDENAR15, UDENAR11, SINDAMANOY, UDENAR4, UDENAR10 y UDENAR14 con promedios entre 6,89 y 7,11 granos por vaina, los cuales mostraron diferencias significativas respecto a las líneas UDENAR 12, UDENAR 16 y UDENAR 17 (5.40, 5.24 y 5.00 granos por vaina). La variedad Andina, mostró una media de 6,73, estadísticamente similar al 91\% de los genotipos evaluados, con diferencias $(p<0,05)$ sobre las líneas UDENAR16 y UDENAR17 que obtuvieron 5,24 y 5,00 granos por vaina. Lo anterior demuestra que entre las líneas con gen afila evaluadas, existen genotipos promisorios para este caracter que logran igualar estadísticamente el promedio de NGV de los testigos no afila Andina y Sindamanoy. Los resultados sugieren que hay independencia entre los genes que controlan la expresión del caracter afila y los que corresponden al número de granos por vaina, pues tener o no la presencia de hojas transformadas en zarcillos por efecto del gen afila no implica una reducción o un aumento en el número de granos por vaina. Al respecto, Tulcán y Castillo (1998) sugieren que el número de granos por vaina es un carácter cuya expresión depende en alto grado de la composición genética del material. El comportamiento de las medias para este rasgo fue similar al reportado por Khan et al. (2013) donde las variaciones estuvieron entre 4,0 y 8,5 granos por vaina.

El NGV, es uno de los componentes de rendimiento más importantes en el mejoramiento genético de arveja para el caso de Colombia, donde más del $90 \%$ de la producción de arveja se destina al mercado de vaina verde. Lo deseable es un número igual o superior a seis granos por vaina, pero la mejora genética es difícil dado 
que hay mecanismos de compensación en los componentes de rendimiento, de tal forma que si se aumentan las semillas por vaina, se puede disminuir el número de vainas por planta o el peso de la semilla (Tiemerman et al., 2004; Ligarreto et al., 2009).

Cuando el NGV se sitúa entre tres y cuatro, se considera bajo, medio entre cinco y seis y alto mayor de seis (Biddle et al., 1988). Según lo anterior, $22,7 \%$ de los materiales genéticos evaluados tuvieron un NGV medio y el 77,3\% alto. Los genotipos que se encontraron en rango alto fueron:
UDENAR1, UDENAR2, UDENAR3, UDENAR4, UDENAR6, UDENAR7, UDENAR8, UDENAR9, UDENAR10, UDENAR11, UDENAR13, UDENAR14, UDENAR15, UDENAR19, UDENAR20, Andina y Sindamanoy, constituyendo un grupo de materiales fenotípicamente deseables para este caracter. En estudios realizados por Rea (2012), los promedios para esta variable fluctuaron entre 6 y 10 granos por vaina mostrando mayor variabilidad para este rasgo, respecto a los observados en la presente evaluación que estuvieron entre 5,00 y 7,11 granos por vaina lo cual se explica por bajo número de progenitores y su similitud genética.

Tabla 2. Comparación de promedios de Tukey para las variables: número de granos por vaina (NGV), peso de vaina verde (PVV), peso de grano en vaina verde (PGVV) y rendimiento (REND)

\begin{tabular}{|c|c|c|c|c|c|c|c|c|}
\hline \multirow{2}{*}{$\begin{array}{l}\text { GENOTIPO } \\
\text { UDENAR15 }\end{array}$} & \multicolumn{2}{|c|}{ NGV } & \multicolumn{2}{|c|}{ PVV } & \multicolumn{2}{|c|}{ PGVV } & \multicolumn{2}{|c|}{ REND } \\
\hline & 7,11 & $\mathrm{a}$ & 6,24 & bcdef & 3,91 & bcdefg & 8,72 & $\mathrm{~b}$ \\
\hline UDENAR11 & 7,06 & $\mathrm{a}$ & 8,04 & abcd & 4,99 & $\mathrm{ab}$ & 7,40 & $\mathrm{~b}$ \\
\hline SINDAMANOY & 7,00 & $\mathrm{a}$ & 8,21 & $a b c$ & 5,26 & $\mathrm{a}$ & 15,26 & $\mathrm{a}$ \\
\hline UDENAR4 & 6,99 & $\mathrm{a}$ & 5,29 & ef & 3,13 & fg & 7,85 & $\mathrm{~b}$ \\
\hline UDENAR10 & 6,97 & $\mathrm{a}$ & 7,24 & abcdef & 4,20 & abcdefg & 7,23 & $\mathrm{~b}$ \\
\hline UDENAR14 & 6,89 & $\mathrm{a}$ & 8,13 & $a b c$ & 5,24 & $\mathrm{a}$ & 9,37 & $a b$ \\
\hline UDENAR1 & 6,80 & $a b$ & 7,46 & abcdef & 4,66 & $a b c$ & 7,29 & $\mathrm{~b}$ \\
\hline ANDINA & 6,73 & $a b$ & 8,95 & $\mathrm{a}$ & 5,06 & $a b$ & 11,50 & $a b$ \\
\hline UDENAR20 & 6,71 & $a b$ & 7,93 & abcde & 4,35 & abcde & 8,78 & $a b$ \\
\hline UDENAR13 & 6,71 & $a b$ & 5,40 & def & 3,66 & cdefg & 6,46 & $\mathrm{~b}$ \\
\hline UDENAR19 & 6,42 & $a b c$ & 8,77 & $a b$ & 4,29 & abcdef & 6,00 & $b$ \\
\hline UDENAR3 & 6,42 & $a b c$ & 6,00 & cdef & 3,53 & cdefg & 6,86 & $\mathrm{~b}$ \\
\hline UDENAR7 & 6,37 & $a b c$ & 5,95 & cdef & 3,77 & cdefg & 7,88 & $\mathrm{~b}$ \\
\hline UDENAR9 & 6,31 & $a b c$ & 5,29 & ef & 3,44 & defg & 6,97 & $\mathrm{~b}$ \\
\hline UDENAR2 & 6,29 & $a b c$ & 7,46 & abcdef & 4,51 & abcd & 8,99 & $a b$ \\
\hline UDENAR6 & 6,22 & $a b c$ & 5,93 & cdef & 3,08 & g & 6,47 & $\mathrm{~b}$ \\
\hline UDENAR8 & 6,11 & $a b c$ & 5,18 & $\mathrm{f}$ & 3,17 & efg & 6,33 & $\mathrm{~b}$ \\
\hline UDENAR18 & 5,73 & $a b c$ & 7,26 & abcdef & 3,71 & cdefg & 6,49 & b \\
\hline UDENAR5 & 5,70 & $a b c$ & 6,22 & bcdef & 3,65 & cdefg & 7,35 & $\mathrm{~b}$ \\
\hline UDENAR12 & 5,40 & $\mathrm{bc}$ & 7,95 & abcd & 4,55 & abcd & 7,45 & $\mathrm{~b}$ \\
\hline UDENAR16 & 5,24 & $\mathrm{c}$ & 6,00 & cdef & 3,40 & defg & 7,77 & $\mathrm{~b}$ \\
\hline UDENAR17 & 5,00 & $\mathrm{c}$ & 6,33 & abcdef & 3,42 & defg & 7,54 & $\mathrm{~b}$ \\
\hline Tukey & 1,428 & & 2,66 & & 1,19 & & 6,514 & \\
\hline
\end{tabular}




\section{Peso de vaina verde (PVV)}

Teniendo en cuenta que las 20 líneas evaluadas provienen de cruzamientos entre variedades volubles mejoradas de vaina grande, con genotipos que aportaron el gen afila (ILS3575, ILS3568 y Dove) los cuales son de vaina pequeña, entonces la obtención de líneas afila que conserven el tamaño de vaina comercial es uno de los objetivos de la selección. Las líneas con gen afila que se destacaron para este carácter fueron UDENAR19, UDENAR14 y UDENAR11 con promedios entre 8,77 y 8,04 g las cuales logran igualar estadísticamente a las variedades Andina y Sindamanoy $(8,95$ y 8,21 g). Al respecto, Valencia y Timaná (2013), reportaron para las variedades Andina y Sindamanoy promedios de 7,36 g y 8,06 g, respectivamente.

Independientemente de la presencia del gen afila en las líneas evaluadas, la variación observada en el PVV, depende de la constitución genética que para este rasgo tienen los parentales que dieron origen a estas líneas. Al revisar la genealogía de estas líneas, se observó que en UDENAR19 uno de sus parentales fue la variedad San Isidro, mientras que para UDENAR14 y UDENAR11 uno de sus parentales fue la variedad Sindamanoy. Estos progenitores contribuyeron a aportar genes que influyeron positivamente en el tamaño de la vaina. En contraste, los padres que aportaron el gen afila y que fueron usados para la obtención de estas líneas como son Dove e ILS3575, presentan vainas pequeñas que contribuyen negativamente a la expresión de este carácter. En consecuencia, no fue posible superar a los testigos en el peso de vaina verde, pero sí acercarse a sus promedios.

Desde el punto de vista comercial, el peso de vaina verde incluyendo el grano en fresco, es muy importante para los compradores de arveja, quienes buscan y pagan mejor las vainas grandes y de mayor peso porque las asocian con mayor tamaño y peso de los granos. Sin embargo, hay agricultores y comerciantes que sostienen que cuando las variedades de arveja se siembran en las zonas altas por encima de 2800 $\mathrm{msnm}$, las vainas pueden presentar mayor peso debido al aumento en el grosor de sus valvas pero que ello no significa mayor tamaño y peso de sus granos.

En tales circunstancias, es necesario determinar hasta que altura puede sembrarse las diferentes variedades de arveja sin perder sus características óptimas de vaina y grano.

\section{Peso de grano verde por vaina (PGVV)}

La variedad Sindamanoy y la línea UDENAR14 con promedios de 5.26 y $5.24 \mathrm{~g}$, superaron al $54,5 \%$ de las líneas evaluadas que mostraron un PGVV inferior a 3,92 g (Tab. 2). Por otra parte, la variedad Andina y la línea UDENAR11 (5,06 y $4.99 \mathrm{~g}$ ) superan al 50\% de las líneas evaluadas que tuvieron promedios por debajo de 3,78 g. El $36,4 \%$ del material evaluado con promedios entre 5,24 y 4,20 g (UDENAR1, UDENAR2, UDENAR10, UDENAR11, UDENAR12, UDENAR14, UDENAR19 y UDENAR20) igualaron estadísticamente a la variedad Sindamanoy. Además, la línea UDENAR15 (3,91 g) no presentó diferencias estadísticas con la variedad Andina. Esta variable es importante puesto que representa la parte útil para el consumo de esta leguminosa. En general son muy apreciadas en el mercado las variedades con mayor peso de grano por vaina, porque se asocian con mayor número de granos por vaina y con granos de mayor tamaño.

\section{Rendimiento (REND)}

Los rendimientos oscilaron entre 15,26 y $6,00 \mathrm{t}$ ha $^{-1}$. Las líneas con gen afila UDENAR14, UDENAR2, UDENAR20 con promedios entre 9,37 y 
8,78 igualaron estadísticamente a las variedades comerciales Andina y Sindamanoy de follaje normal (Tab. 2), mientras que las demás líneas evaluadas mostraron un rendimiento estadísticamente igual a la variedad Andina $(\mathrm{P}<0,05)$. La variedad Sindamanoy $\left(15,26 \mathrm{t} \mathrm{ha}^{-1}\right)$ superó al $77,27 \%$ de los genotipos evaluados. Tanto en la variedad Sindamanoy como en Andina, el excesivo follaje y abundantes ramas laterales obliga a los agricultores a utilizar el sistema de tutorado vertical y el de encanastillado u horizontal, para evitar el acame y el deterioro de las vainas por el ataque de patógenos como Ascochyta pisi y Colletotrichum pisi, lo cual representa un incremento en los costos de producción por tutorado que alcanza hasta el 52\% de los costos totales del cultivo (Eraso y Cuarán, 2010). Encontrar líneas de arveja, menos exuberantes que las variedades Sindamanoy y Andina, pero con rendimientos similares, contribuirá a reducir los costos de producción por tutorado. Las líneas UDENAR14, UDENAR2, UDENAR20, que lograron rendimientos estadísticamente similares a Sindamanoy y Andina $(\mathrm{P}<0,05)$, pueden seleccionarse para futuras investigaciones en las que se verifique su comportamiento en sistemas de tutorado más sencillos, debido a la presencia en ellas del gen afila que favorece el mantenimiento de plantas erguidas, al permitir mejor agarre de sus zarcillos sobre los hilos de polipropileno que se usan como tutores. Además, entre las plantas contiguas de un mismo surco los zarcillos se juntan dándole mayor firmeza al sistema de tutorado e impidiendo el acame. Por otra parte en estas líneas afila, es conveniente hacer estudios de densidades de plantas para explotar mejor su potencial productivo, considerando que al reducir el follaje es posible establecer más plantas por unidad de área.

La reducción del follaje puede disminuir el ataque de enfermedades foliares tal como lo afirman Mera et al. (1996); Giaconi (2004); Gonzáles
(2001); Prieto (2011). Por otra parte, es posible que al reducir su área foliar, estas líneas puedan soportar una mayor densidad de siembra lo cual puede contribuir a aumentar el rendimiento.

Se encontró un elevado coeficiente de variación para este rasgo $(26,00 \%)$, similar al reportado por Muñoz (2013) que fue de 27,63\% al evaluar 15 cultivares de arveja entre los cuales habían volubles y no volubles y al obtenido por Sultana et al. (2002) con un valor de 37,84\% según el cual se debe al efecto ambiental.

\section{Análisis de correlación}

Los coeficientes estimados de correlación fenotípica $(\mathrm{rF})$, genética $(\mathrm{rG})$ y ambiental $(\mathrm{rE})$ están registrados en la Tabla 3. Los caracteres que acusaron alto grado de correlación fenotípica $(\mathrm{p}$ $<0,05)(\mid$ ri $\mid>0,50)$ fueron: PVV x PGVV $(0,88)$, PVV x REND $(0,70)$ y PGVV $x \operatorname{REND}(0,63)$; Por otra parte se obtuvieron altas correlaciones genotípicas para PVV x PGVV $(0,91)$, PVV x REND $(0,88)$ y PGVV x REND $(0,76)$, mientras que las correlaciones ambientales ( $\mathrm{rE}$ ) significativas estuvieron dadas por NGV $x$ PVV $(0,65), N G V \times$ PGVV $(0,73)$, PVV x PGVV $(0,59)$.

Los coeficientes de correlación genética que acusaron mayores valores en magnitud $(|\mathrm{rG}|>0,50)$ con respecto al rendimiento de arveja en vaina verde (REND) fueron: peso de vaina verde (PVV: 0,88$)$ y peso de grano verde por vaina (PGVV: 0,76). Estas correlaciones implican que dichos caracteres pueden ser importantes indicadores de rendimiento en poblaciones de alta variabilidad y revela que la selección de uno o ambos rasgos se puede traducir en un rendimiento superior, lo cual tiene explicación desde el punto de vista genético por la existencia de una acción conjunta de genes que gobiernan o determinan dichos rasgos tal como lo afirman Pandey y Gritton (1975). 
Según Bedell (2006), la correlación genotípica puede ser debida a la acción pleiotrópica de un gen o debida al ligamiento de genes. El mismo autor indica que si la asociación entre dos rasgos (ya sea positiva o negativa) sigue siendo la misma en poblaciones parentales así como en poblaciones segregantes, significa que la asociación es resultado de la pleiotropía.

Este tipo de correlación es más importante para lograr el mejoramiento genético de un caracter mediante la selección de otro carácter genéticamente correlacionado. Las correlaciones genotípicas encontradas presentan resultados similares a los reportados por Rasaei et al. (2011); Kosev y Mikic (2012).

Para el número de granos por vaina (NGV) no se encontró correlación fenotípica respecto al rendimiento (REND). Sin embargo, al descartar el efecto ambiental se obtuvo una correlación genética significativa baja entre estos dos caracteres $(0,37)$ lo cual indica que en un reducido grado el número de granos por vaina estuvo asociado con el rendimiento; resultado similar al obtenido por Avci y Ceyhan (2006). Por su parte, Sultana et al. (2002), reportaron elevados coeficientes de correlación genotípica $(0,97)$ y fenotípica $(0,89)$ entre número de granos por vaina y rendimiento, al igual que en las investigaciones de Avci y Ceyhan (2001), donde el valor de correlación fenotípica permitió tomar al número de granos por vaina como criterio de selección y cuyos resultados se pueden ver en poblaciones de Ceyhan et al., 2008, 2012.

Las diferencias en los coeficientes de correlación entre componentes de rendimiento, reportados por distintos autores, pueden relacionarse con diferencias en variabilidad genética y condiciones ambientales. Es importante considerar que las correlaciones fenotípicas tienen poco valor práctico, son riesgosas y pueden conllevar a errores, pues incluyen la asociación entre caracteres tanto de naturaleza genética como ambiental (Ceballos, 2003).

El coeficiente de correlación ambiental es debido a los efectos ambientales y a las causas genéticas no aditivas (Dabhholkar, 1999). En otras palabras, está asociado con un error de varianza. Siendo de menor importancia, por no ser heredable ni estable. Si el coeficiente de correlación genotípica es mayor que el coeficiente de correlación fenotípica, entonces existe una fuerte asociación genética entre esos dos caracteres, pero el valor fenotípico puede ser disminuido por la interacción significativa del ambiente (Bedell, 2006). Lo anterior fue observable en las correlaciones PVV x PGVV, PVV x REND y PGVV x REND (Tab. 3).

Tabla 3. Correlaciones fenotípicas $\left(\mathrm{r}_{\mathrm{F}}\right)$, genéticas $\left(\mathrm{r}_{\mathrm{G}}\right)$ y ambientales $\left(\mathrm{r}_{\mathrm{E}}\right)$ para 4 caracteres en arveja ( $P$. satioum): Número de granos por vaina (NGV), peso de vaina verde (PVV), peso de grano verde por vaina (PGVV) y rendimiento (REND)

\begin{tabular}{lllll}
\hline & $\mathbf{r}^{\prime} \mathbf{s}$ & PVV & PGVV & REND \\
\hline \multirow{4}{*}{ NGV } & $\mathrm{r}_{\mathrm{f}}$ & 0,28 & $0,44^{*}$ & 0,33 \\
& $\mathrm{r}_{\mathrm{G}}$ & 0,22 & $0,40^{*}$ & $0,37^{*}$ \\
& $\mathrm{r}_{\mathrm{E}}$ & $0,65^{*}$ & $0,73^{*}$ & $0,26^{*}$ \\
\hline \multirow{3}{*}{ PVV } & $\mathrm{r}_{\mathrm{f}}$ & & $0,88^{*}$ & $0,70^{*}$ \\
& $\mathrm{r}_{\mathrm{G}}$ & & $0,91^{*}$ & $0,88^{*}$ \\
& $\mathrm{r}_{\mathrm{E}}$ & & $0,59^{*}$ & 0,14 \\
\hline \multirow{4}{*}{ PGVV } & $\mathrm{r}_{\mathrm{f}}$ & & & $0,63^{*}$ \\
& $\mathrm{r}_{\mathrm{G}}$ & & & $0,76^{*}$ \\
& $\mathrm{r}_{\mathrm{E}}$ & & & $0,26^{*}$ \\
\hline
\end{tabular}

*Correlación significativo $(\mathrm{P}<0,05)$ 
En algunas circunstancias, los coeficientes fenotípicos y genotípicos entre dos caracteres pueden ser similares, indicando que no existe efecto significativo de los factores ambientales sobre el nivel de asociación real de los caracteres en estudio (Espitia et al., 2008). En otros casos como en la correlación NGV x PVV de la presente investigación en donde la correlación fenotípica es ligeramente superior a la genética, se puede deducir un efecto del medio, el cual se confirma por el mayor valor del coeficiente de correlación ambiental $(0,65)$.

\section{Análisis de sendero}

Los resultados del análisis de coeficiente de sendero indican que para alcanzar una correlación genotípica entre NGV y REND de 0,37 (Tab. 3), la variable NGV aportó con un efecto directo de 0,32 y las variables PVV y PGVV con unos efectos indirectos de 0,30 y $-0,25$, respectivamente. Es de resaltar que a pesar de que la correlación genotípica entre los dos caracteres (NGVxREND), es relativamente baja, la misma depende en alto grado del efecto directo del NGV (Tab. 4; Fig. 1). Lo anterior no significa que la variable no se deba tener en cuenta en otras investigaciones, puesto que sería muy probable que se destaque en poblaciones de alta variabilidad genética. En contraste, Kumar et al. (2013), obtuvieron un efecto bajo pero negativo $(-0,23)$, mientras que Sonali et al. (2009), encontraron que esta variable se debe tener en cuenta para los programas de mejoramiento de arveja en sus materiales evaluados debido a su elevado efecto directo sobre la variable rendimiento.

Tabla 4. Descomposición de las correlaciones genotípicas $\left(r_{\mathrm{g}}\right)$ en efectos directos (diagonal) e indirectos (fuera de la diagonal), mediante análisis de sendero, para el rendimiento de arveja, en función del número de granos por vaina (NGV), peso de vaina verde (PVV) y peso de grano verde por vaina (PGVV).

\begin{tabular}{lccc}
\hline Variables & NGV & PVV & PGVV \\
\hline NGV & 0,32 & 0,30 & $-0,25$ \\
PVV & 0,07 & 1,4 & $-0,59$ \\
PGVV & 0,13 & 1,27 & $-0,64$ \\
\hline
\end{tabular}

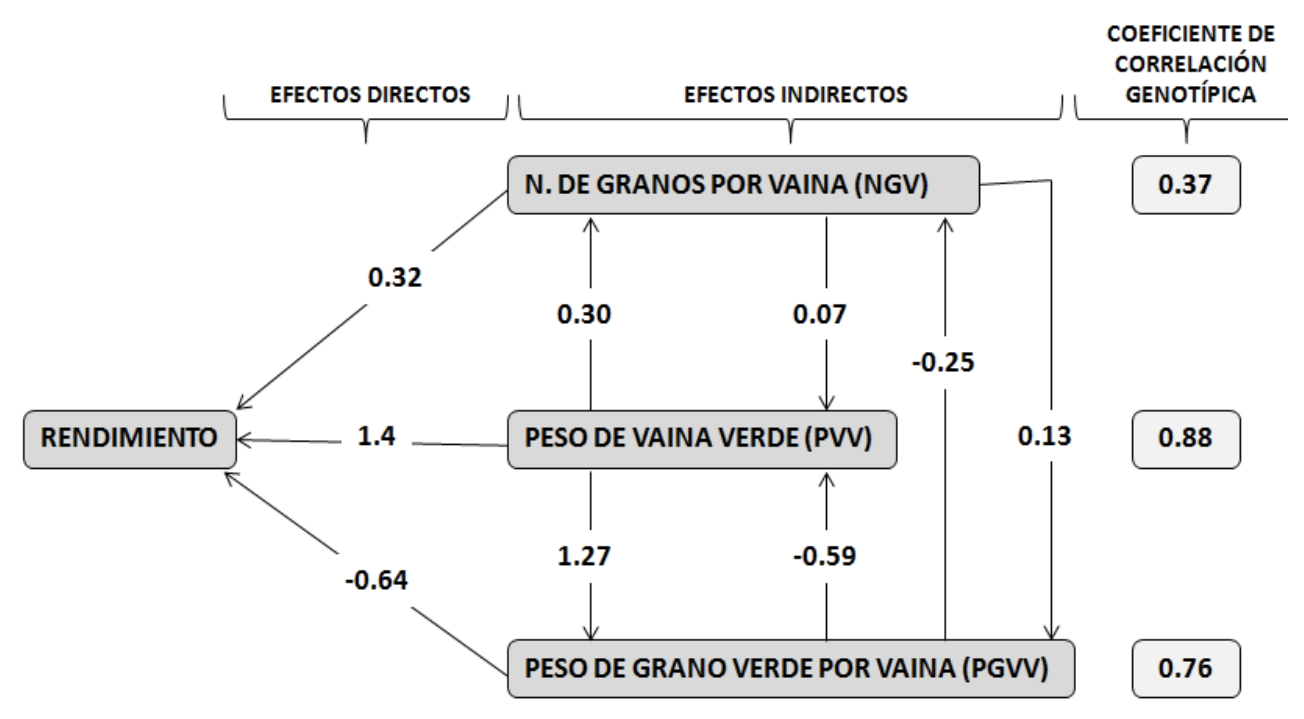

Figura 1. Diagrama de sendero mostrando los coeficientes de sendero del rendimiento en líneas de arveja (P. sativum L.) con gen afila. 
Para la correlación genotípica entre PVV y REND que fue de 0,88 , la variable PVV contribuyó con un efecto directo de 1,4, mientras que los caracteres NGV y PGVV aportaron los efectos indirectos de 0,07 y $-0,59$. Lo anterior sugiere que el efecto directo de PVV fue determinante para lograr la alta correlación de ésta variable con el rendimiento (REND). Por lo tanto, es claro que el peso de vaina verde (PVV) es una variable a través de la cual es posible realizar la selección de los genotipos por rendimiento.

La correlación genotípica entre PGVV y REND fue de 0,76 (Tab. 3). El efecto directo de PGVV que contribuyó a esta correlación fue de -0,64 y los efectos indirectos de las variables NGV y de PVV que aportaron a la misma, fueron de 0,13 y de 1,27. El valor negativo del efecto directo de PGVV $(-0,64)$ enfrentado al alto valor positivo del efecto indirecto aportado por $\operatorname{PVV}(1,27)$, sugiere que la correlación genotípica obtenida entre PGVV y REND $(0,76)$ está más soportada en los efectos indirectos en especial el de PVV, en consecuencia, no es posible utilizar la variable PGVV para la selección por rendimiento.

A pesar de que con el análisis de sendero, es posible identificar variables a través de las cuales se puede realizar selección indirecta por rendimiento o por otro rasgo de interés, es necesario tener en cuenta que los resultados obtenidos en esta investigación, solo son aplicables al grupo de líneas evaluadas, puesto que ellas fueron seleccionadas y no representan una muestra aleatoria de los genotipos de arveja existentes en una colección de amplia base genética.

Los resultados de correlaciones genéticas encontrados entre las variables peso de vaina verde (PVV) y peso de grano verde por vaina (PGVV) frente a rendimiento (REND) $(0,88$ y 0,76 respectivamente), sugieren que es posible seleccionar para rendimiento (REND), a través de las varia- bles PVV y PGVV, en tal sentido se espera que los genotipos UDENAR11, UDENAR12, UDENAR14, UDENAR19 y UDENAR20 que lograron igualar al menos a uno de los testigos Andina y/o Sindamanoy, los igualen también en rendimiento, lo cual se confirmó en la comparación de promedios realizada para esta variable (Tab. 2).

\section{CONCLUSIONES}

Las líneas con gen afila evaluadas no mostraron efecto en las variables altura de planta (ALT) y número de vainas por planta (NVP) respecto a los testigos de hojas normales Andina y Sindamanoy.

El peso de vaina verde (PVV) y el peso de grano por vaina verde (PGVV) presentaron alta correlación genética con el rendimiento (REND).

Las líneas con gen afila UDENAR11, UDENAR12, UDENAR14, UDENAR19 y UDENAR20, sobresalieron para los rasgos peso de vaina verde (PVV) y peso de grano por vaina verde (PGVV). En el análisis de sendero el peso de la vaina verde presentó el mayor efecto directo en la correlación genotípica entre los componentes de rendimiento y el rendimiento en fresco.

Las líneas con gen afila evaluadas, mostraron rendimientos similares a la variedad Andina y constituyen una opción en la búsqueda de alternativas para mejorar la competitividad del cultivo de arveja a través de sistemas de tutorado más simples.

\section{AGRADECIMIENTOS}

Los autores agradecen a la Facultad de Ciencias Agrícolas de la Universidad de Nariño, al Grupo de Investigación en Cultivos Andinos de la 
Universidad de Nariño y al Centro Internacional de Producción Limpia - Lope SENA Regional Nariño por el apoyo recibido para el desarrollo de la presente investigación.

\section{BIBLIOGRAFÍA}

AVCI, M.A y CEYHAN, E. 2001. Relation among seed yield and some morphological characteristics of pea cultivars (Pisum sativum L.) sown in various sowing dates under central Anatolian. S.U. Ziraat Fakultesi Dergisi. 15:173 - 183.

AVCI, M. y CEYHAN, E. 2006. Correlations and genetic analysis of pod characteristics in pea (Pisum sativum L.). Asian Journal of Plant Sciences.5:1 - 4 .

BEDELL, P. 2006. Tree breeding for genetic improvement of tropical tree species. Allied Publishers Private Limited. India. 201p.

BIDDLE, A.J., KNOTT, C.M. y GENT, G.P. 1988. The PGRO pea growing handbook. Sixth edition. Processors and Growers Research Organization, England. $264 \mathrm{p}$

CEBALLOS, H. 2003. Genética cuantitativa y fitomejoramiento. Universidad Nacional de Colombia Sede Palmira. 524 p.

CEYHAN, E., KAHRAMAN, M.K. y KARADAS. 2008. Line $X$ tester analysis in pea (Pisum sativum L.): Identification of superior parents for seed yield and its components. Africa Journal Biotechnology. 7(16):2810 - 2817.

CEYHAN, E., KAHRAMAN, M.K. y KARADAS. 2012. Stability analysis on seed yield and its components in peas. Agriculture Science Bulgarian. 18:905 - 911.

CHECA, O. 2014.Investigación para el mejoramiento de la tecnología de producción de arveja (Pisum sativum L.) en el departamento de Nariño. En: http://www.ucc.edu.co/ pasto/prensa/2014/Documents/1Resumen_ Proyecto_L\%C3\%ADneas\%20Arveja.pdf.; consulta: octubre, 2014.

COHEN, L. y HOLLIDAY, M. 1996. Practical statistics for students. First edition, Globe graphics. London. 362 p.

COUSIN R, A., MESSAGER y VINGERE, A. 1985. Breeding for yield in combining peas, pp. 15-129. En: HEBBLETHWAITE, P.D. HEATH, M.C. and DAWKINS, T.C.K. The pea crop: A basis for improvement. First edition. Butterworths. Nottinghamshire. 5:56 - 78.

CRUZ, D. 2004. Programa genes. Versão Windows. Aplicativo computacional em genética e estatística. Universidade Federal de Viçosa. En: www.ufv.br/dbg/genes/genes.htm.; consulta: enero, 2014.

DABHHOLKAR, A. 1999. Elements of bio metrical genetics, (Revised And Enlarged Edition). Concept Publishing Company. New Delhi. 493 p.

DEPARTMENT OF HORTICULTURAL SCIENCE. 1999. Vegetable cultivar descriptions for North America. University Raleigh. North Carolina State. 970 p.

DJORDJEVIC, R., MARKOVIC, Z., PESIC, V. y DJINOVIC, I. 2001.The influence of "afaf" genes on pea (Pisum sativum L.) pod formation agricultural Research Institute "Serbia", Smederevska Palanka Yugoslavia. Center for Vegetable Crops. 26(2):135 - 140.

ENCUESTA NACIONAL AGROPECUARIA. 2014. En: https://www.dane.gov.co/ files/investigaciones/agropecuario/sipsa/ Semana_04ene_10ene_2014.pdf; consulta: octubre, 2014. 
ENGELS, J., HAWKES, J. y WOREDE, M. 1991. Plant Genetics Resources of Ethiopia. First published. Cambridge University Press. New York. 383 p.

ERASO, A. y CUARÁN, S. 2010. Evaluación de 15 líneas promisorias de arveja arbustiva (Pisum sativum L.) en cinco municipios de la zona sur del departamento de Nariño. Tesis Ingeniero Agrónomo, Facultad de Ciencias Agrícolas. Universidad de Nariño. Pasto. 28 p.

ESPITIA, M., ARAMÉNDIZ, H y CADENA, J. 2008. Correlaciones y análisis de sendero en algodón (Gossypium hirsutum L.) en el Caribe Colombiano. Revista Facultad Nacional de Agronomía. 61(1):4325 - 4335.

FALCONER, D. 1986. Introducción a la genética cuantitativa. Segunda edición. Compañía Editorial Continental. México. 383 p.

FEDERACIÓN NACIONAL DE CULTIVADORES DE CEREALES (FENALCE). 2010. El cultivo de arveja historia e importancia. Disponible en: http://www.fenalce.org/arch_public/arveja93. pdf; consulta: febrero, 2014.

FIERRO, R. 2012. Estabilidad de 20 líneas de arveja voluble (Pisum sativum L).en cinco municipios del sur del departamento de Nariño. Tesis de Magister en Ciencias Agrarias Universidad de Nariño. Maestría en Ciencias Agrarias. Área de Énfasis Producción de cultivos. San Juan de Pasto. 54 p.

GARSON, G. D. 2012. Path Analysis. Asheboro, North Carolina: Statistical Associates Publishers. $52 \mathrm{p}$.

GARZON, M. y GASCA, H. 1990. Comportamiento de dos variedades de arveja (Pisum sativum L.) para uso industrial bajo dos sistemas de siembra y cuatro densidades en las condiciones de la Sabana de Bogotá. Tesis Facultad de Agro- nomía, Universidad Nacional de Colombia. Bogotá. $177 \mathrm{p}$.

GIACONI, V. 2004. Cultivo de hortalizas. 15 ed. Santiago de Chile: Editorial Universitaria. 120 p.

GOLDENBERG, J. B. 1965. “Afila” a new mutation in pea (Pisum sativum L.). Boletín Genética 1: 27-28.

GONZÁLES, M. 2001. Interacción genotipo x ambiente en guisante proteaginoso (Pisum sativum L.). Tesis doctoral. Universidad de Valladolid: Departamento De Producción Vegetal y Silvopascicultura. $272 \mathrm{p}$.

HAZARD, B. 2005. Statistical methods for health Care Research. Philadelphia: Lippincott Williams \& Wilkins. 1:377 p.

HEDLEY, C.L., y AMBROSE, M. J. 1981. Designing "leafless" plants for improving the dried pea crop. Advances in Agronomy. 34:225 - 227.

INSTITUTO TÉCNICO Y DE GESTIÓN AGRÍCOLA DE NAVARRA (ITGA). 2003. Guisante proteaginoso en Navarra, campaña 20032004.12 p.

JANNINK, J.L., LIEBMAN, M. y MERRICK, L.C. 1996. Biomass production and nitrogen accumulation in pea, oat and vetch green manure mixtures. Agronomy Journal. 88:231 - 240.

KHAN, T.N., RAMZAN, G. JILLANI, G. y MEHMOOD. 2013. Morphological performance of peas (Pisum sativum L.) genotypes under rainfed conditions of Potowar region. Journal of Agricutural Research. 51:51 - 60.

KOSEV, V y MIKIĆ, A. 2012. Short communication. Assessing relationships between seed yield components in spring-sown field pea (Pisum sativum L.) cultivars in Bulgaria by correlation and path analysis. Spanish Journal of Agricultural Research. 10(4):1075 - 1080. 
KUJALA, V.1953. Felderbse, beiwelcher die ganz Blattspreite in Ranken umgewandelt ist. Archivum.100 Societatis Zoologica e Botanica e Fennicae "Vanamo", 8:44 - 45.

KUMAR, B., KUMAR, A. y ROOPA, L. 2013. Selection strategy for seed yield and maturity in field pea (Pisum satioum L.).African Journal of Agricultural Research. 8(44):5411 - 5415.

LIGARRETO, M., GUSTAVO, A., OSPINA, H. y ANDRÉS, R. 2009. Análisis de parámetros heredables asociados al rendimiento y precocidad en arveja voluble (Pisum sativum L.) tipo Santa Isabel. Agronomía Colombiana. 27(3):333 - 339.

MARTIN, I., TENORIO, J. y AYERBE, L. 1994. Yield, Growth, and water use of conventional and semi-leafless peas in semi-arid environments. Crop Science.34:76 - 83.

MARX, G.A. 1987. A suite of mutants that modify pattern formation in pea leaves. Plant Molecular Biology Reporter. 5:311 - 335.

MERA, M., LEVÍO, J., ALCALDE, J. MORALES, M. y GALDAMES, R. 1996. Brisca-INIA, primera variedad de arveja áfila obtenida en Chile. Agricultura técnica (Chile). 56 (4):282 - 286.

MERA, M., J.M. ALCALDE, y S. FERRADA. 1998. Arvejas para congelado en el sur: Sorprendente potencial de rendimiento. Tierra Adentro. 28:23 - 25.

MERA, M., KEHR, E., MEJIAS, J., IHL, M. y BIFANI, V. 2007. Arvejas (Pisum sativum L.) de vaina comestible sugarsnap: Antecedentes y comportamiento en el sur de Chile. Agricultura Técnica. 67(4):343 - 352.

MIHAILOVIC, V. 2008. Componentes de rendimiento de grano afila $(a f)$ líneas de guisantes forrajeros (Pisum satioum L.). Instituto de Cultivos y Hortalizas, NoviSad, Serbia. 98 p.

MOSES, E. 2006. A user's Guide to Path Analy- sis. (Ilustrada) University Press of America. Lanham.171 p.

MUÑOZ, S.R. 2013. Evaluación agronómica de quince cultivares de arveja (Pisum Sativum L.), mediante el apoyo de investigación participativa con enfoque de género en la estación experimental del austro Bullcay. Tesis presentada como requisito parcial para obtener el título de lingeniero Agrónomo. Facultad de Recursos Naturales, Escuela Superior Politécnica De Chimborazo. Riobamba. 118 p.

PANDEY, S. y GRITTON, E.T. 1975. Genotypic and phenotypic variances and correlations in peas. Crop Science. 15: 353 - 356.

PRIETO, G. 2011. El Cultivo de Arveja. AERINTA Arroyo Seco. 13 p.

PROHENS.J y NUEZ, T. 2007. Vegetables II: Liliaceae, Solanaceae, and Umelliferae. Springer Science and Bussines Media. Handbook of plant breeding. Springer. New York. 365 p.

RASAEI, A., EGHBAL, M., GHOBADI, $\mathrm{M}$ y NIYA, A. 2011.The study of traits correlation and path analysis of the grain yield of the peas in semi-dry conditions in Kermanshah.University Kermanshah. Irán.4 p.

REA, M. 2012. Evaluación de la aclimatación y rendimiento de 15 cultivares de arveja (Pisum sativum L.) a campo abierto, en Macaji, Canton Riobamba, Provincia de Chimborazo. Escuela Superior Politécnica Chimborazo, Facultad De Recursos Naturales. Escuela De Ingeniería Agronómica. Riobamba-Ecuador. 84 p.

SAÑUDO, B. CHECA, O. y ARTEAGA, G. 1999. Manejo agronómico de leguminosas En Zonas Cerealistas. Primera edición. Produmedios, Colombia. $97 \mathrm{p}$.

SARUTAYOPHAT, T. 2012. Correlation and path coefficient analysis for yield and its com- 
ponents in vegetable soybean. Sonkglanakarin Journal of science and technology. Bangkok. 34(3):273 - 277.

SINGH, M., HARI, D y BISTH, S. 2013. Genetic and genomic resources of grain legume improvement. First edition. ELSEVIER INSIGHTS. London. $322 \mathrm{p}$.

SONALI G, NIRMLA C, y SAROJ D. 2009. Genetic variability, correlation and path analysis studies in pea (Pisum sativum L.). Crop Research (Hisar). 38(1-3):179 - 183.

SULTANA, Z., A., ISLAM, K. M. A., HASAN MITUAND,M. K. y MIAN.A. K. 2002. Genetic variability And character association in garden pea (Pisum sativum L. spp. Hortense) genotypes. Dept of Genetics and Plant Breeding Bangabandhu Sheikh Mujibu Rahman Agricultural University. Bangladesh. 9 p.

TIEMERMAN, V., MILLS, G., FREW, T., BUTLER, T., MCCALLUM, J., S., MURRAY, C., WHITFIELD, A., RUSELL, A y WILSON, D. 2004. Linkage mapping of QTLs for seed yield, yield components and developmental traits in pea $(\mathrm{Pi}$ sum sativum L.). En: $4^{\text {th }}$ International Crop Science Congress. Brisbane, Australia.

TULCÁN, G. y CASTILLO, C. 1998. Efecto de la labranza y aplicación de herbicidas en el manejo de malezas en el cultivo de arveja (Pisum sativum L.) en el municipio de Pasto departamento de Nariño. Tesis Ingeniero Agrónomo. Universidad de Nariño: Facultad de Ciencias Agrícolas. Área Agronomía.

VALENCIA, A y TIMANÁ, Y. 2013. Evaluación de 20 líneas de arveja (Pisum sativum L.) y su reacción al complejo de Ascochyta. Revista de Ciencias Agrícolas. 29(2):2256 - 2273.

WANG, F., FU, J., DONG L. y ZHU, Y. 2003. Tendril inheritance in semi-leafless pea and its utilization in breeding. YiChuan. 25(2):18 p. 